\title{
Chylous Ascites in a Patient with Sepsis Caused by Bilateral Pneumonia
}

\author{
Yong Dae Lee, M.D., Young Hyun Lee, M.D., Ph.D., and Hye Sook Choi, M.D., Ph.D. \\ Department of Internal Medicine, Dongguk University College of Medicine, Gyeongju, Korea
}

Chylous ascites is a rare form of ascites characterized by milky peritoneal fluid rich in triglycerides due to the accumulation of chyle in the peritoneal cavity. This affliction occurs as a result of a disruption of lymph flow associated with traumatic injury or obstruction of the lymphatic system. There are various causes of chylous ascites, such as lymphatic anomalies, malignancy, cirrhosis, infection, trauma, surgery, and nephrotic syndrome. We report a rare case of an 81-year-old male with sepsis caused by bilateral pneumonia who presented with chylous ascites.

Key Words: chylous ascites; hypoalbuminemia; nephrotic syndrome; pneumonia; sepsis.

Chylous ascites (CA) is a rare form of ascites characterized by the accumulation of lipid-rich, milk-like lymphatic fluid in the peritoneal cavity.[1] An elevated triglyceride (TG) level is therefore important in diagnosing CA. CA develops secondary to traumatic injury or obstruction of the lymphatics, causing the leakage of chyle through the walls of dilated lymphatic vessels or a lymphoperitoneal fistula.[2,3] CA is caused by diverse conditions, including abdominal malignancy, lymphatic anomalies, liver cirrhosis and nephrotic syndrome.[2-4]

There has been no case report of CA developed in a patient with pneumonia and accompanying sepsis. In the present case, we describe for the first time CA developed in an elderly male patient undergoing intensive treatment with mechanical ventilation and antibiotics for pneumonia and accompanying sepsis. His CA was treated conservatively with fasting, total parenteral nutrition (TPN), subcutaneously injected octreotide, albumin infusion and continuous drainage of the fluid. We also reviewed the related literature.

Received on September 2, 2013 Revised on April 5, 2014

Accepted on April 28, 2014

Correspondence to: Hye Sook Choi, Department of Internal Medicine, Dongguk University College of Medicine, 87 Dongdae-ro, Gyeongju 780-350, Korea

Tel: +82-54-770-8586, Fax: +82-54-770-8671

E-mail: maxymus72@hanmail.net

* No potential conflict of interest relevant to this article was reported.

\section{Case Report}

An 81-year-old man presented to the emergency department with dyspnea and a three-day history of fever, cough and sputum. The patient had a medical history of hepatitis B and had been taken care of in a convalescent hospital for two months due to dementia. On admission, his vital signs showed a blood pressure of $120 / 70 \mathrm{mmHg}$, a pulse of 92 beats per minute, a respiration rate of 34 breaths per minute and a body temperature of $38.7^{\circ} \mathrm{C}$. The patient had bilateral lung crackles at auscultation, but no abdominal distention or edema. His chest X-ray demonstrated consolidation of both lungs (Fig. 1).

Laboratory findings on admission were as follows: white blood cell count $4,160 / \mu$ l (neutrophils $86.9 \%$, lymphocytes $9.3 \%$ ), hemoglobin $11.7 \mathrm{~g} / \mathrm{dl}$, platelet count $158,000 / \mu \mathrm{l}$, protein $4.5 \mathrm{~g} / \mathrm{dl}$, albumin $2.1 \mathrm{~g} / \mathrm{dl}$, glucose $99 \mathrm{mg} / \mathrm{dl}$, aspartate aminotransferase (AST) $53 \mathrm{IU} / \mathrm{L}$, alanine aminotransferase (ALT) 15 IU/L, total bilirubin $0.91 \mathrm{mg} / \mathrm{dl}$, blood urea nitrogen (BUN) 24 $\mathrm{mg} / \mathrm{dl}$, creatinine $1.2 \mathrm{mg} / \mathrm{dl}$, creatine phosphokinase (CK) 240 IU/L, lactate dehydrogenase (LDH) 256 IU/L, prothrombin time (PT) 11.6 seconds, international normalized ratio of prothrombin time (PT INR) 0.97, lactic acid $5.14 \mathrm{mM} / \mathrm{L}$, C-reactive protein (CRP) $16.9 \mathrm{mg} / \mathrm{dl}$ and erythrocyte sedimentation rate 44 $\mathrm{mm} / \mathrm{h}$. On arterial blood gas analysis (ABGA), $\mathrm{pH}$ was 7.48, $\mathrm{PCO}_{2} 24.8 \mathrm{mmHg}, \mathrm{PO}_{2} 30.8 \mathrm{mmHg}, \mathrm{HCO}_{3}{ }^{-} 18.1 \mathrm{mM} / \mathrm{L}$ and 
$\mathrm{SaO}_{2} 60.3 \%$. The serologic test for hepatitis $\mathrm{B}$ and $\mathrm{C}$ viruses by enzyme immunoassay showed positive for hepatitis B surface antigen, negative for hepatitis B surface antibody, negative for hepatitis B envelope antigen, positive for hepatitis B envelope antibody and negative for antibody to hepatitis $\mathrm{C}$ virus. The pneumococcal urinary antigen test was positive, and a sputum culture grew Streptococcus pneumoniae.

Once the patient was transferred to the intensive care unit (ICU), mechanical ventilation and antibiotics were used for the treatment of his pneumonia and accompanying respiratory fail-

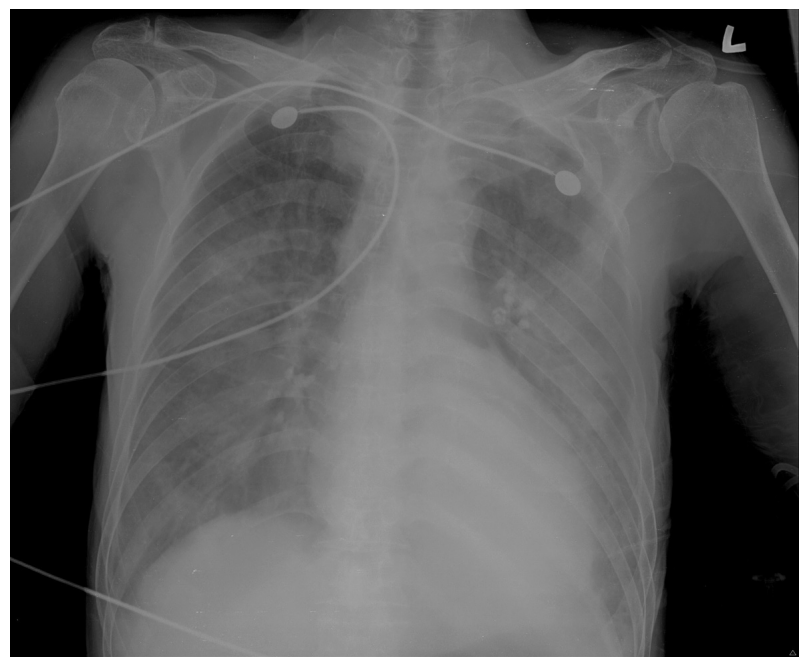

Fig. 1. Initial portable chest X-ray (anteroposterior view) shows diffuse haziness in both lung fields.
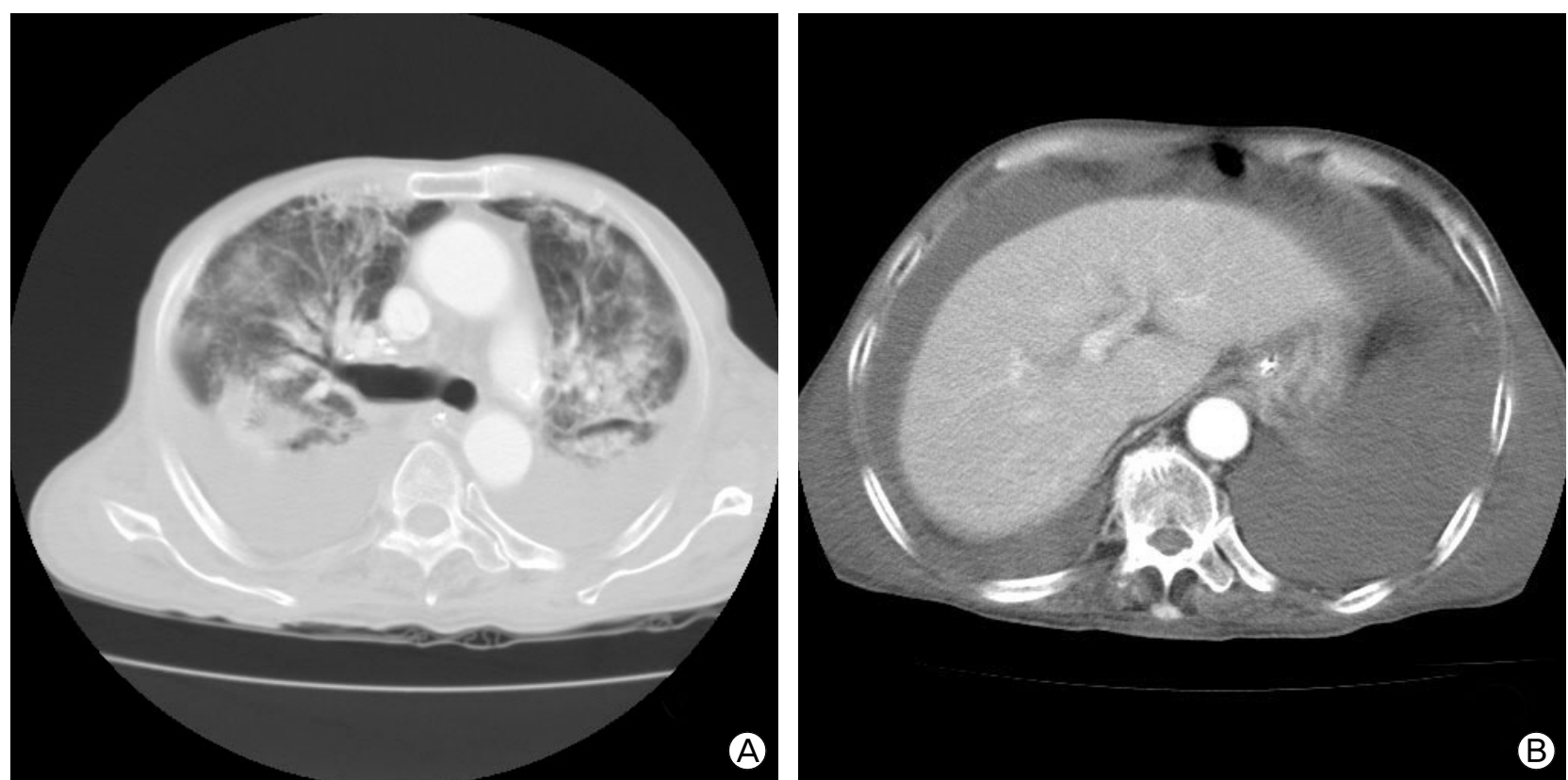

Fig. 2. (A) Chest computed tomography on hospital day 32 shows peribronchial and subpleural consolidation with ground-glass opacity in both lungs and bilateral pleural effusions. (B) It also shows a large amount of ascites in the abdominal cavity. ure and severe sepsis. Albumin was also infused to reverse hypoalbuminemia, and diuretics were used to treat edema developed after admission. While antibiotics were administered according to the susceptibility, sputum culture results revealed extended- spectrum $\beta$-lactamase-producing Klebsiella pneumoniae, multidrug-resistant Acinetobacter baumannii, and multidrug-resistant Pseudomonas aeruginosa. Meropenem and colistin were therefore administered.

At 32 days after admission, mechanical ventilation was still continued, and the patient had a blood pressure of $130 / 80 \mathrm{mmHg}$, a pulse of 100 beats per minute and intermittent fever hovering around $38^{\circ} \mathrm{C}$. While his clinical condition did not improve, the patient developed generalized edema and abdominal distention, calling for computed tomography (CT) scan. The chest $\mathrm{CT}$ revealed peribronchial and subpleural consolidation with ground-glass opacity in upper and middle lobes of both lungs, bilateral pleural effusions and large-volume ascites (Fig. 2).

At that time, laboratory findings showed the following results: white blood cell count $6,640 / \mu$ l (neutrophils $86.2 \%$, lymphocytes $8.6 \%$ ), hemoglobin $7.4 \mathrm{~g} / \mathrm{dl}$, platelet count $63,000 / \mu \mathrm{l}$, protein $3.5 \mathrm{~g} / \mathrm{dl}$, albumin $1.7 \mathrm{~g} / \mathrm{dl}$, AST $67 \mathrm{IU} / \mathrm{L}$, ALT $54 \mathrm{IU} / \mathrm{L}$, CK 497 IU/L, LDH 701 IU/L, PT 13.7 seconds, PT INR 1.21, CRP $4.73 \mathrm{mg} / \mathrm{dl}$, total cholesterol $63 \mathrm{mg} / \mathrm{dl}$ and TG $107 \mathrm{mg} / \mathrm{dl}$. Hypoalbuminemia was still persistent. The ABGA revealed that his blood $\mathrm{pH}$ was 7.53, $\mathrm{PCO}_{2} 33.3 \mathrm{mmHg}, \mathrm{PO}_{2} 68.3 \mathrm{mmHg}$, total bilirubin $0.79 \mathrm{mg} / \mathrm{dl}$, BUN $28 \mathrm{mg} / \mathrm{dl}$, creatinine $0.7 \mathrm{mg} / \mathrm{dl}$, 


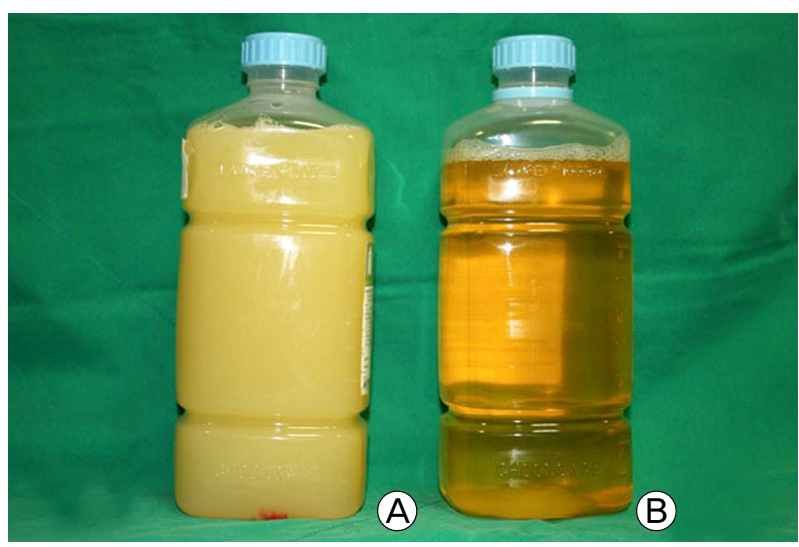

Fig. 3. (A) The ascitic fluid, which was drained through a pig-tail catheter, shows a turbid and milky appearance. (B) The pleural fluid shows a serous effusion.

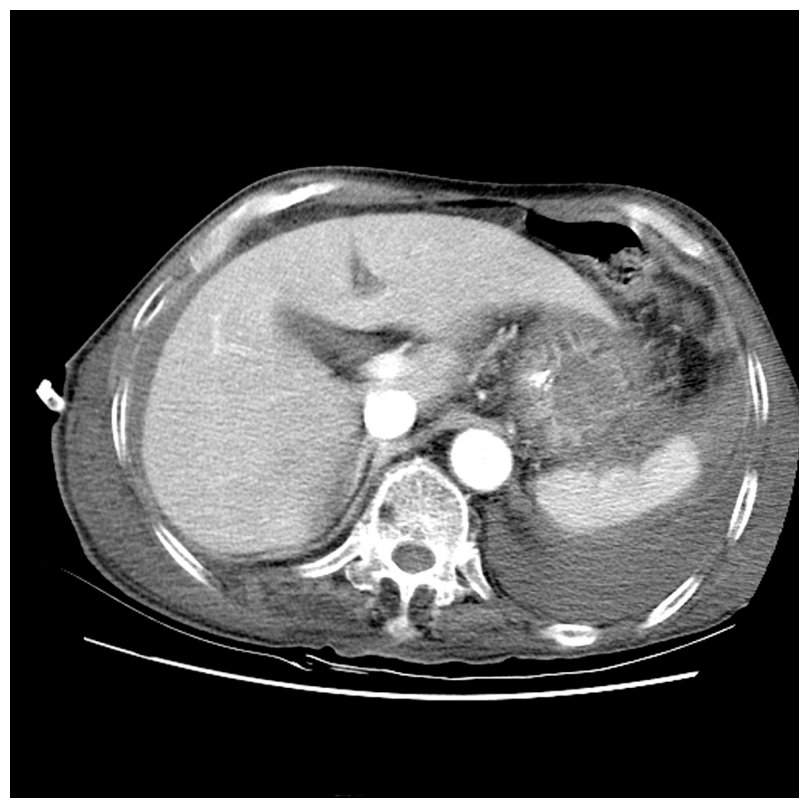

Fig. 4. Abdominal computed tomography on hospital day 33 shows no other significant findings except for much less ascites after draining intraperitoneal fluid with a pig-tail catheter placed in the peritoneal cavity.

$\mathrm{HCO}_{3}{ }^{-} 29.2 \mathrm{mM} / \mathrm{L}$ and $\mathrm{SaO}_{2} 95.5 \%$. The ascitic fluid obtained by paracentesis was milky in gross appearance (Fig. 3a), and the

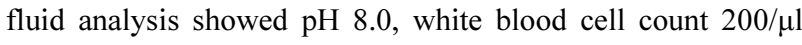
(neutrophils $38 \%$, lymphocytes $58 \%$ ), protein $0.8 \mathrm{~g} / \mathrm{dl}$, albumin $0.6 \mathrm{~g} / \mathrm{dl}$, glucose $125 \mathrm{mg} / \mathrm{dl}$, total cholesterol $12 \mathrm{mg} / \mathrm{dl}$, TG 163 $\mathrm{mg} / \mathrm{dl}, \mathrm{LDH} 125 \mathrm{IU} / \mathrm{L}$, amylase $31 \mathrm{IU} / \mathrm{L}$ and adenosine deaminase (ADA) $9 \mathrm{IU} / \mathrm{L}$, leading to the diagnosis of CA. In addition, the results of ordinary bacterial and mycobacterial cultures, polymerase chain reaction for Mycobacterium tuberculosis and cytology were all negative. The pleural fluid by thoracentesis was serous in appearance (Fig. 3b), showing pH 8.0, white blood cell count 2,980/ $\mu \mathrm{l}$ (neutrophils $95 \%$, lymphocytes $5 \%$ ), protein $1.5 \mathrm{~g} / \mathrm{dl}$, albumin $1.0 \mathrm{~g} / \mathrm{dl}$, glucose $109 \mathrm{mg} / \mathrm{dl}$, LDH $300 \mathrm{IU} / \mathrm{L}$ (normal serum range 101-218 IU/L), amylase $25 \mathrm{IU} / \mathrm{L}$ and ADA $11 \mathrm{IU} / \mathrm{L}$. The pleural effusion was therefore confirmed as a parapneumonic effusion that is exudative and neutrophil-dominant.

CA was treated with TPN in combination with fasting, drainage was steadily performed through a pig-tail catheter that was inserted into the abdomen, and administration of octreotide, somatostatin analog, was started. A post-drainage abdominal CT imaging showed reduced accumulation of ascites but no other findings such as cirrhosis, mass and portal vein thrombosis (Fig. 4). A portable transthoracic echocardiogram also revealed no hemodynamic abnormalities such as heart failure or pericarditis.

Octreotide was injected subcutaneously at a dose of $0.1 \mathrm{mg}$ every 12 hours for 5 days. After four days of starting the treatment, CA turned serous, the drainage volume decreased from $1,000 \mathrm{cc}$ to $400 \mathrm{cc}$ per day and the TG level in ascitic fluid decreased to $23 \mathrm{mg} / \mathrm{dl}$. Since then, the amount of drainage did not reduce much, but the ascitic fluid did not appear milky and its TG content was low. For a few days immediately after CA disappeared, serum albumin levels moved in the range of 1.6 to 2.1 $\mathrm{g} / \mathrm{dl}$ without showing a significant increase despite albumin replacement. However, the patient died from sepsis around one month after the occurrence of CA, and there was no follow-up examination.

\section{Discussion}

CA results from diverse conditions, including malignancy, lymphatic disorder, cirrhosis, infection, surgery, trauma, altered hemodynamics, abdominal radiation therapy, acute and chronic pancreatitis and nephrotic syndrome.[2-4] The leading cause of CA in adults is malignancy, particularly lymphoma.[5] Malignancy and cirrhosis are common causes of CA in western countries, while infectious etiologies such as tuberculosis and filariasis commonly cause $\mathrm{CA}$ in developing countries.[3] In addition, hemodynamic abnormalities such as constrictive pericarditis, right heart failure, dilated cardiomyopathy and rheumatic valvular disease can cause chylous ascites by increasing pressure within the lymphatic vessels. $[3,6]$ The pathophysiological mechanisms by which CA develops in pericarditis are that excessive capillary filtration caused by elevated hepatic venous pressure increases the production of lymph and that lymphatic drainage is impeded by chronic venous hypertension.[6] Cirrhosis causes increased hepatic lymph formation,[3] and CA in a cirrhotic pa- 
tient appears to be associated with the lymph leakage resulting from increased intra-abdominal pressure combined with degenerative changes of the splanchnic lymphatic vessels.[4] CA can be caused by other conditions inducing portal hypertension such as portal vein thrombosis.[6,7] Abdominal aortic surgery is the leading cause of postoperative CA.[5] The mechanism of CA formation in nephrotic syndrome is not clearly understood, but is likely to be associated with hypoalbuminemia-induced bowel edema leading to chyle leakage or malabsorption.[8] Acute chylous peritonitis is mostly idiopathic and usually diagnosed by laparotomy in the presence of signs of acute peritonitis.[9]

In investigating the causes of CA, serum-ascites albumin gradient (SAAG) should be calculated to decide whether or not the ascites is related to portal hypertension.[3] In our patient, the SAAG was a borderline value of $1.1 \mathrm{~g} / \mathrm{dl}$, blood cell counts during the hospital course showed thrombocytopenia and anemia, which resulted from sepsis, frequent blood sampling, nutritional deficiency and other factors, and mild elevation of liver enzymes are more likely due to sepsis rather than liver disease.

CA is a milky, alkaline, odorless, sterile fluid and triglyceride levels in CA are typically 2-8 times higher than those in plasma.[6] CA has TG content of more than $200 \mathrm{mg} / \mathrm{dl}$, although some researchers used the cutoff of $110 \mathrm{mg} / \mathrm{dl}$.[3] Smears of CA stained with Sudan III show fat globules and white blood cells with a predominance of lymphocytes.[5] In this case, ascitic lymphocytes were $58 \%$ of leukocytes and TG content was 163 $\mathrm{mg} / \mathrm{dl}$, which exceeds the cutoff of $110 \mathrm{mg} / \mathrm{dl}$ and is 1.52 times higher than $107 \mathrm{mg} / \mathrm{dl}$ in plasma. It is however presumed that the administration of albumin for hypoalbuminemia and diuretics for edema control affected laboratory data on blood, pleural effusion and ascites.

Hypoalbuminemia is commonly caused by malnutrition and liver and kidney diseases. The patient had hypoalbuminemia (albumin $2.1 \mathrm{~g} / \mathrm{dl}$ ) but had no edema at the time of admission. In addition, proteinuria was negative on urinalysis a few times, confirming the absence of nephrotic syndrome. The patient's hypoalbuminemia might have resulted from nutritional deficiency in the convalescent hospital where the patient stayed before admission, but it is more likely that sepsis is responsible for persistent hypoalbuminemia. The mechanisms of hypoalbuminemia in patients with sepsis are reduced liver synthesis, accelerated catabolism and increased leakage into the interstitium due to enhanced vascular permeability.[10]

Abdominal CT is useful in identifying lymph nodes and masses within the abdomen and in deciding the localization and extent of CA particularly when thoracic duct injury is suspected after surgery or trauma. Imaging studies such as lymphangiography and lymphoscintigraphy aid in identifying abnormal retroperitoneal lymph nodes, leakage from dilated lymphatic vessels, fistula formation and opening of the thoracic duct.[3]

Conservative treatment options for $\mathrm{CA}$ include therapeutic paracentesis, a medium-chain TG-based diet, TPN, somatostatin, etc. $[3,5,6]$ The treatment of underlying conditions may decrease ascites and improve symptoms, but a high-protein and low-fat diet with medium-chain TGs would be effective as the initial therapy when the cause of CA has not been identified or CA do not respond to the treatment for underlying disease. Long-chain TGs are excluded from the diet because they are transported as chylomicrons into the lymphatic vessels of the bowel, whereas medium-chain TGs are absorbed into the intestinal cells and turned into free fatty acids and glycerol, which are then delivered through the portal vein to the liver.[3] A low-fat diet based on medium-chain TG is effective in treating chylous fistula by suppressing chyle formation and flow.[5] If the diet does not work, fasting and TPN can be used. Even though the mechanism of somatostatin in the treatment of CA is not well known, somatostatin reduces the amount of lymph circulation in the major lymphatic vessels, suppresses lymph excretion via somatostatin receptors in the wall of the intestine, and may contribute to a reduction in the production of lymph fluid.[1,11] The use of somatostatin is recommended in the early stages before any invasive treatment starts because it has effects on fistula closure. If CA does not respond to conservative management, invasive treatment, such as peritoneovenous shunting or surgery, is necessary.[5] Surgical approach can be effective particularly when CA occurs following a surgery and when CA is caused by tumor or congenital abnormalities. Lymphangiography or lymphoscintigraphy before surgery is useful to identify the anatomic location of a leak and the presence of fistula.[3]

In this report, we described CA developed in an elderly male patient having pneumonia and accompanying sepsis and hypoalbuminemia without his pneumonia improving despite the treatment with mechanical ventilation and antibiotics in the ICU. The contribution of the chyle leak to the whole ascites resolved with conservative management, including fasting, TPN, subcutaneously injected octreotide, albumin infusion and continuous drainage. The follow-up examination did not take place because the patient died from sepsis. We however concluded that CA was likely caused by hypoalbuminemia, which was persistent along with sepsis, similarly to the mechanism of CA in nephrotic syndrome. It is believed that a transudative fluid shift occurred as a result of volume overloading and hypoalbuminemia 
during the sepsis treatment and therefore the dilution effect, such as elevation of SAAG, came about both in ascites and in pleural effusion. Presumably, that is why the volume of ascites reduced but drainage still continued after conservative management. Although other possibilities cannot be ruled out, it appears that the reduction of $\mathrm{CA}$ volume with no significant increase of the plasma albumin level was greatly influenced by other conservative treatment options on top of albumin administration. The abdominal CT did not suggest any clues for the underlying cause of $\mathrm{CA}$, and lymphangiography and lymphoscintigraphy were not performed in this patient because the contribution of CA to the whole ascites disappeared. The portable echocardiography also revealed no hemodynamic abnormalities that could cause $\mathrm{CA}$

CA combined with pneumonia and accompanying sepsis has not been reported to date. Here we report a rare case of CA developed in sepsis caused by pneumonia and suggest a possible development of CA when ascites occurs in patients undergoing intensive care for sepsis.

\section{References}

1) Yildirim AE, Altun R, Can S, Ocal S, Akbaş E, Korkmaz M, et al: Idiopathic chylous ascites treated with total parenteral nutrition and octreotide. A case report and review of the literature. Eur J Gastroenterol Hepatol 2011; 23: 961-3.
2) Browse NL, Wilson NM, Russo F, al-Hassan H, Allen DR: Aetiology and treatment of chylous ascites. Br J Surg 1992; 79: $1145-50$

3) Cárdenas A, Chopra S: Chylous ascites. Am J Gastroenterol 2002; 97: 1896-900.

4) Steinemann DC, Dindo D, Clavien PA, Nocito A: Atraumatic chylous ascites: Systematic review on symptoms and causes. J Am Coll Surg 2011; 212: 899-905.e1-4.

5) Leibovitch I, Mor Y, Golomb J, Ramon J: The diagnosis and management of postoperative chylous ascites. J Urol 2002; 167(2 Pt 1): 449-57.

6) Aalami OO, Allen DB, Organ CH Jr: Chylous ascites: a collective review. Surgery 2000; 128: 761-78.

7) Leong RW, House AK, Jeffrey GP: Chylous ascites caused by portal vein thrombosis treated with octreotide. J Gastroenterol Hepatol 2003; 18: 1211-3.

8) You ZH, Yang AH, Yang WC, Huang TP, Lin CC: Chylous ascites. NDT Plus 2010; 3: 315.

9) Vettoretto N, Odeh M, Romessis M, Pettinato G, Taglietti L, Giovanetti M: Acute abdomen from chylous peritonitis: A surgical diagnosis. Case report and literature review. Eur Surg Res 2008; 41: 54-7.

10) Gatta A, Verardo A, Bolognesi M: Hypoalbuminemia. Intern Emerg Med 2012; 7 Suppl 3: S193-9.

11) Huang Q, Jiang ZW, Jiang J, Li N, Li JS: Chylous ascites: Treated with total parenteral nutrition and somatostatin. World J Gastroenterol 2004; 10: 2588-91. 